\title{
Editorial: Improving cancer chemotherapy through pharmacogenomics: a research topic
}

\author{
Luis A. Quiñones ${ }^{1 *}$ and Kuen S. Lee ${ }^{2}$ \\ 'Laboratory of Chemical Carcinogenesis and Pharmacogenetics, Clinical and Molecular Pharmacology Program, Faculty of \\ Medicine, Instituto de Ciencias Biomédicas, University of Chile, Santiago, Chile, ${ }^{2}$ Department of Surgery, Hospital del \\ Salvador, University of Chile, Santiago, Chile
}

Keywords: pharmacogenomics, pharmacogenetics, polymorphism, genetic, oncology, antineoplastic agents

OPEN ACCESS

Edited by:

Ulrich M. Zanger,

Dr. Margarete Fischer-Bosch-Institute of Clinical Pharmacology, Germany

Reviewed by:

Kathrin Klein,

University of Tübingen, Germany

${ }^{*}$ Correspondence:

Luis A. Quiñones,

lquinone@med.uchile.cl

Specialty section:

This article was submitted to

Pharmacogenetics and

Pharmacogenomics,

a section of the journal

Frontiers in Genetics

Received: 30 April 2015

Accepted: 17 May 2015

Published: 03 June 2015

Citation:

Quiñones LA and Lee KS (2015)

Editorial: Improving cancer chemotherapy through

pharmacogenomics: a research topic.

Front. Genet. 6:195.

doi: 10.3389/fgene.2015.00195
It is well known that the efficacy and safety of chemotherapeutic drugs show substantial individual and/or population variability that can be explained, to a great extent, by genetic factors in pharmacokinetics and pharmacodynamics (Quiñones et al., 2008; Roco et al., 2012; Izar et al., 2013; Kessler et al., 2014; Patel, 2015; Unger et al., 2015). In the context of anticancer drugs, polymorphisms in genes encoding drug-metabolizing enzymes, drug transporters and drug targets influence the pharmacokinetics and pharmacodynamics, have been shown to affect clinical outcomes (Binkhorst et al., 2015; Newman et al., 2015). Several chemotherapeutic agents can be more harmful to normal tissues than the targeted tumor, and their use may result in tumor cell resistance, toxicity and occasionally secondary neoplasia. Current practices for the dosing of therapeutic agents could be improved through the understanding of gene variation that impacts the life of the drug inside the body. In order to better predict a patient's predisposition to treatment complications and poor outcomes, it is essential to consider all candidate loci influencing response to the chemotherapeutic agents. This requires better understanding of the metabolic pathways for activation or inactivation of these drugs, drug interactions, age and gender sensitivities, and the impact of ethnicity and environmental factors. As an added benefit, a more rational use of expensive treatment drugs, together with actions to minimize patient toxic events and their consequences, would dramatically reduce medical costs.

Pharmacogenomics is an emerging field focused on genetic variations relevant to drug action (Evans and Relling, 2004; Maxmen, 2011; Mayer, 2014; Zhou et al., 2015). Solid data concerning allele variants, haplotypes and their effects on gene expression, applied to the use of chemotherapy regimens' design and dosing has the potential to enhance treatment outcomes. Some of the potential polymorphic candidate genes include the CYP isozymes, transferases, dehydrogenases, deaminases, reductases, $\mathrm{ABC}$ transporters, drug receptors and DNA repair enzymes (Agundez, 2004; Bruhn and Cascorbi, 2014; Roco et al., 2014).

With this Research Topic we aim to present an updated overview of the current trends in the experimental genetic approaches worldwide, and discuss future directions in cancer pharmacotherapy. The scope of this issue is to collect evidence to conceive chemotherapy tailored to each patient.

In this special edition we have five manuscripts: four reviews and one original article. Filipski et al. (2014) discuss the use of observational studies, as well as the use adaptive trials and next generation sequencing to achieve the required level of evidence for clinical implementation. They propose to move the research field toward retrospective analyses, large population studies using Electronic Medical Records, and mechanism-based evidence to propel progress in the field. In addition, embracing new methods, such as next generation sequencing 
and pharmacogenomic adaptive clinical trials, will enrich our knowledge about tumor biology, and change the clinical approaches for cancer treatment. They highlight that up-to-date pharmacogenomic studies tend to focus on either somatic or germline mutations in isolation, but to truly optimize clinical care both genomes need to be integrated into clinical decision making. Agúndez et al. (2014) review the development of clinical practice recommendations or guidelines for the clinical use of pharmacogenomics data. They suggest that this is an essential issue for improving drug therapy, particularly for drugs with high toxicity and/or narrow therapeutic index, such as anticancer agents. The few available clinical practice guidelines for translating Pharmacogenomics are insufficient for its application in the use of anticancer therapy. The application of available guidelines, further implementation with clinical feedback, plus a combination of genomics and phenomics is urgently required. Roco and co-workers explore the possibility for prediction of the efficacy and safety of cisplatin treatment using a pharmacogenomic approach, and focus primarily on DNA reparation and Glutathione S-Transferases (GSTs) enzymes. They conclude that there is a potential utility for pharmacogenetics in therapeutic schemes employing cisplatin. This could be addressed through collaborative efforts to study molecular characteristics of drug treatment in order to develop a genetic panel for this context.

Finally, T. Ishikawa's mini-review and the original article of Zocche et al. (2015) describe the use of two relevant genes in oncology as prognostic biomarkers. Ishikawa (2014) focuses on genetic polymorphism in the NRF2 gene, especially in the future

\section{References}

Agundez, J. A. (2004). Cytochrome P450 gene polymorphism and cancer. Curr. Drug Metab. 5, 211-224. doi: 10.2174/1389200043335621

Agúndez, J. A., Esguevillas, G., Amo, G., and García-Martín, E. (2014). Clinical practice guidelines for translating pharmacogenomic knowledge to bedside. Focus on anticancer drugs. Front. Pharmacol. 5:188. doi: 10.3389/fphar.2014.00188

Binkhorst, L., Mathijssen, R. H., Jager, A., and van Gelder, T. (2015). Individualization of tamoxifen therapy: much more than just CYP2D6 genotyping. Cancer Treat Rev. 41, 289-299. doi: 10.1016/j.ctrv.2015. 01.002

Bruhn, O., and Cascorbi, I. (2014). Polymorphisms of the drug transporters ABCB1, ABCG2, ABCC2 and ABCC3 and their impact on drug bioavailability and clinical relevance. Expert Opin. Drug Metab. Toxicol. 10, 1337-1354. doi: 10.1517/17425255.2014.952630

Evans, W. E., and Relling, M. V. (2004). Moving towards individualized medicine with pharmacogenomics. Nature 429, 464-468. doi: 10.1038/nature 02626

Filipski, K. K., Mechanic, L. E., Long, R., and Freedman, A. N. (2014). Pharmacogenomics in oncology care. Front. Genet. 5:73. doi: $10.3389 /$ fgene. 2014.00073

Ishikawa, T. (2014). Genetic polymorphism in the NRF2 gene as a prognosis marker for cancer chemotherapy. Front. Genet. 5:383. doi: $10.3389 /$ fgene. 2014.00383

Izar, B., Rotow, J., Gainor, J., Clark, J., and Chabner, B. (2013). Pharmacokinetics, clinical indications, and resistance mechanisms in molecular targeted therapies in cancer. Pharmacol. Rev. 65, 1351-1395. doi: 10.1124/pr.113. 007807 for individualized cancer lung cancer treatment. The author supports the role of this gene as a mediator of cancer cell proliferation and drug resistance, and highlight the $-617 \mathrm{C}>\mathrm{A}$ change in the anti-oxidant response element (ARE)-like loci as relevant in the attenuation of the positive feedback loop of transcriptional activation of NRF2, leading to reduced NRF2 protein levels. As a consequence, it is considered cancer cells will become more sensitive to therapy and less aggressive than cancer cells harboring the wild type allele. Therefore, genetic polymorphisms in NRF2 might serve as prognosis markers for cancer therapy. Finally, Zocche et al. (2015) investigated the clinical application and the Global Impact of KRAS mutation patterns in 5-fluorouracil regimens (FOLFOX and FOLFIRI). The study investigated the progression-free survival and overall survival in colorectal cancer patients receiving FOLFOX treatment with respect to KRAS mutations. They conclude that G12D KRAS mutation was significantly associated with a poor prognosis.

We proudly present the current Research Topic and believe it contributes evidence for the better understanding of the genetic basis in individual differences in response to antineoplastic drugs, as well as suggesting potential biomarkers for treatment prognosis.

\section{Acknowledgments}

We would like to thank all the authors for their contributions to this topic and Dr. Jana Stojanova for her critical review of this editorial letter. The research of Dr. LQ is supported by Fondecyt Grant \#1140434.

Kessler, D. A., Austin, R. H., and Levine, H. (2014). Resistance to chemotherapy: patient variability and cellular heterogeneity. Cancer Res. 74, 4663-4670. doi: 10.1158/0008-5472.CAN-14-0118

Maxmen, A. (2011). Pharmacogenomics: playing the odds. Nature 474, S9-S10. doi: $10.1038 / 474$ S9a

Mayer, D. K. (2014). Response to "personalized medicine, genomics, and pharmacogenomics". Clin. J. Oncol. Nurs. 18, 618. doi: 10.1188/14. CJON.618

Newman, E. M., Morgan, R. J., Kummar, S., Beumer, J. H., Blanchard, M. S., Ruel, C., et al. (2015). A phase I, pharmacokinetic, and pharmacodynamic evaluation of the DNA methyltransferase inhibitor 5-fluoro-2'-deoxycytidine, administered with tetrahydrouridine. Cancer Chemother. Pharmacol. 75, 537-546 doi: 10.1007/s00280-0142674-7

Patel, J. N. (2015). Cancer pharmacogenomics: implications on ethnic diversity and drug response. Pharmacogenet. Genomics 25, 223-230. doi: 10.1097/FPC.0000000000000134

Quiñones, L., Rosero, M., Roco, A., Moreno, I., Varela, F., Caceres, D., et al. (2008). Role of Cytochrome P450 enzymes in the metabolism of antineoplasic drugs: present situation and future perspectives. Rev. Med. Chile 136, 1327-1335. doi: 10.4067/S0034-98872008001000015

Roco, A., Cayún, J., Contreras, S., Stojanova, J., and Quiñones, L. (2014). Can pharmacogenetics explain efficacy and safety of cisplatin pharmacotherapy? Front. Genet. 5:391. doi: 10.3389/fgene.2014.00391

Roco, A., Quiñones, L., Agúndez, J. A., García-Martín, E., Squicciarini, V., Miranda, C., et al. (2012). Frequencies of 23 functionally significant variant alleles related with metabolism of antineoplastic drugs in the chilean population: comparison with caucasian and asian populations. Front. Genet. 3:229. doi: 10.3389/fgene.2012.00229 
Unger, F. T., Witte, I., and David, K. A. (2015). Prediction of individual response to anticancer therapy: historical and future perspectives. Cell Mol. Life Sci. 72, 729-757. doi: 10.1007/s00018-014-1772-3

Zhou, Z. W., Chen, X. W., Sneed, K. B., Yang, Y. X., Zhang, X., He, Z. X., et al. (2015). Clinical association between pharmacogenomics and adverse drug reactions. Drugs 75, 589-631. doi: 10.1007/s40265-0150375-0

Zocche, D. M., Ramirez, C., Fontao, F. M., Costa, L. D., and Redal, M. A. (2015). Global impact of KRAS mutation patterns in FOLFOX treated metastatic colorectal cancer. Front. Genet. 6:116. doi: 10.3389/fgene.2015.00116
Conflict of Interest Statement: The authors declare that the research was conducted in the absence of any commercial or financial relationships that could be construed as a potential conflict of interest.

Copyright (๑) 2015 Quiñones and Lee. This is an open-access article distributed under the terms of the Creative Commons Attribution License (CC BY). The use, distribution or reproduction in other forums is permitted, provided the original author(s) or licensor are credited and that the original publication in this journal is cited, in accordance with accepted academic practice. No use, distribution or reproduction is permitted which does not comply with these terms. 